\title{
El modelo cooperativo de sistemas agroalimentarios de innovación: el caso ANECOOP y el sistema citrícola valenciano
}

\author{
J.R. Gallego-Bono y R. Chaves-Avila \\ Departament d'Economía Aplicada. Universitat de València. Facultat d'Economia, Avinguda de Taron- \\ gers s/n, 46022 Valencia (Spain)
}

\begin{abstract}
Resumen
La globalización presiona a los sistemas territoriales de producción a convertirse en sistemas territoriales de producción y de innovación para mantener su competitividad. Pero la heterogeneidad empresarial que presentan estos sistemas dificulta la respuesta a estos desafíos y obliga a que dicha respuesta sea colectiva y a nivel meso-económico. Hasta ahora se ha defendido el liderazgo de algunas empresas privadas y otros actores intermediarios, como las asociaciones empresariales, como vehículos de este proceso, pero sin apenas estudiar el papel de las cooperativas en el mismo. En este sentido, el objetivo del artículo es doble. En primer lugar, se trata de conceptualizar de forma novedosa la capacidad de transformación de los sistemas territoriales de producción que tienen las cooperativas en sistemas de innovación. La combinación de un enfoque evolucionista con el enfoque de la gobernanza y el emprendimiento colectivo de la economía social hace emerger una tercera vía (entre la vía privada y la pública) propiamente cooperativa de transformación de estos sistemas a través de un modelo cooperativo de sistema de innovación capaz de generar nuevas meso-reglas. En segundo lugar, y sobre la base de la realización de 218 entrevistas en profundidad con diversos actores del sistema citrícola valenciano, se evidenciará cómo la cooperativa de segundo grado ANECOOP ha liderado la conformación de un modelo cooperativo de sistema de innovación. Surgen así nuevas rutinas y formas de organización vía interacción y redefinición de las comunidades de práctica y epistémicas en respuesta a las nuevas exigencias productivo-comerciales de conocimiento científico-tecnológico.
\end{abstract}

Palabras clave: Cooperativas, sistemas territoriales de producción y de innovación, meso-reglas, emprendimiento colectivo, política de innovación.

\begin{abstract}
The cooperative model of agri-food innovation systems: ANECOOP and the Valencian citrus industry system

Globalisation brings pressure on regional production systems to become regional production and innovation systems in order to maintain their competitiveness. However, the heterogeneity of the companies in these systems makes it difficult to respond to these challenges and obliges the response to be collective and mesoeconomic. To date, there has been a defence of the leadership of some private companies and other intermediaries such as business associations as vehicles of this process, but the part that cooperatives play in it has barely been studied. This paper therefore pursues a double aim. Firstly, it un-
\end{abstract}

* Autor para correspondencia: Rafael.Chaves@uv.es http://dx.doi.org/10.12706/itea.2015.023 
dertakes a novel conceptualisation of the cooperatives' capacity for transforming regional production systems into innovation systems. Combining an evolutionary approach with that of governance and the collective entrepreneurship of the social economy brings to light a truly cooperative "third way" (between private and public) to transform these systems through a cooperative model of an innovation system that is capable of generating new meso-rules. Secondly, based on 218 in-depth interviews with different players in the Valencian citrus industry, it shows how the second-tier cooperative ANECOOP has led the shaping of a cooperative model of an innovation system. As a result, in response to the new demands of growers, packers and marketers for scientific and technical knowledge, new routines and forms of organisation have arisen through the interaction and redefinition of the communities of practice and the epistemic communities.

Key words: Cooperatives, regional production and innovation systems, meso-rules, collective entrepreneurship, innovation policy.

\section{Introducción}

Con la globalización, la liberalización de los mercados y la proliferación de normas y estándares para los productos, acaecidas en las últimas décadas, la capacidad de competir de empresas y sectores económicos enteros se ha visto alterada significativamente. Hoy son competidores globales las grandes corporaciones empresariales y los clusters, también denominados distritos industriales y sistemas territoriales de producción de pequeñas y medianas empresas, quedando condenadas a la marginalidad aquellas empresas que no han logrado integrarse en alguno de los dos anteriores. En el centro de esta estructural mega-transformación económica, uno de los elementos más relevantes para que clusters y corporaciones mantengan y desarrollen capacidades competitivas radica en su capacidad de generar innovación de modo sostenido. Y esta innovación no sólo se concreta en la aparición de nuevos productos agroalimentarios y nuevas técnicas de cultivo o de proceso, sino en una amplia gama de ámbitos que van desde la capacidad de hacer frente a las plagas y enfermedades hasta el dar respuestas óptimas a las necesidades de calidad, gustos y seguridad alimentaria que exige la demanda, como son la trazabilidad o la agricultura ecológica.
La literatura que ha abordado el papel de la innovación en los clusters, y más exactamente, que ha analizado los procesos de transformación de estos sistemas territoriales de producción en sistemas productivos innovadores ha sido prolífica en los últimos años (Lundvall, 1992; Cooke, 2003; Carbonara, 2004; Corò y Micelli, 2007; Gallego, 2008; Temblay et al., 2012; Torre y Wallet, 2013). El cómo incorporar mayores dosis de conocimiento científico en los procesos de innovación, el cómo urdir un entramado adecuado de relaciones con empresas y universidades, centros tecnológicos y otras entidades del entorno peri-productivo funcionales con tales procesos, así como en lograr que ambos sean sostenidos en el tiempo, han constituido los principales desafíos científicos y prácticos. También existe consenso en esta literatura respecto al nivel de análisis para aprehender el ámbito y las exigencias de este proceso de transición hacia la innovación sostenida, el cual no es micro sino meso-económico.

A pesar de representar una parte muy significativa, tanto cuantitativa como cualitativa, de los sectores agroalimentarios de los países desarrollados, el papel de las cooperativas en los procesos de innovación y en la conformación de sistemas territoriales de producción e innovación ha sido escasamente abordado. Cierta literatura sí ha abordado el rol 
de las cooperativas en la construcción de grupos empresariales y holdings, en busca de eficiencias organizativas y ligadas al tamaño o también su papel en la transmisión de las políticas agrarias (Nilsson y Van Dijk, 1997; Julià et al., 2012; Meliá y Marí, 2013; Arcas y Hernández, 2013; Iliopoulos, 2013), pero escasamente en el ámbito que aquí se acomete. Por ello, el objetivo central de este artículo consiste en abordar la función desplegada por las cooperativas en la conformación de sistemas territoriales agroalimentarios innovadores. La hipótesis de partida es que la importancia de las cooperativas no es tanto cuantitativa cuanto cualitativa en estos procesos y sistemas de innovación, y ello tanto por la posición que ocupan estas entidades como por las funciones que desempeñan y por el modo genuino de gobernanza que les caracteriza y que las hace acreedoras de una posición clave, la cual justifica, por sí sola, que regulaciones gubernamentales recientes (como las Entidades Asociativas Prioritarias aprobadas por el Gobierno de España) ${ }^{1}$ otorguen un papel crucial a las cooperativas. En otras palabras, en los sistemas agroalimentarios las cooperativas de segundo grado son simultáneamente el motor de este emprendimiento colectivo y un vehículo privilegiado de la política de innovación. Esta hipótesis se basa en que el nivel meso de una economía es el motor de cambio a través del conocimiento y la innovación que propicia, y en que las cooperativas de segundo grado desempeñan un papel esencial en la organización de este espacio meso-económico, conformando el modelo cooperativo de sistema de innovación (Cooperative Regional Innovation System).

La perspectiva teórica utilizada en este trabajo está basada en las aportaciones realizadas en los últimos años desde el enfoque económico evolucionista e institucionalista, que sitúa en el plano meso-económico el motor del cambio y de la innovación, y desde el enfoque socioeconómico del emprendimiento colectivo, cooperativo y de la economía social, que considera la gobernanza y la innovación social como centros de su atención investigadora. Esto último permitirá aprehender el papel de las cooperativas en la conformación de una visión común y una estrategia colectivas que son esenciales para desarrollar y difundir en el tejido empresarial un tipo de aprendizaje relativamente más orientado y coordinado que el tradicional en los sistemas territoriales de producción.

La aportación cooperativa a los procesos de innovación y a la conformación de sistemas territoriales de producción e innovación se analizará a partir de una realidad concreta: el sistema citrícola valenciano donde cooperativas punteras como $\mathrm{ANECOOP}^{2}$ son buques insignia y constituyen la pieza clave del Cooperative Regional Innovation System del sector citrícola valenciano.

\section{Sistemas territoriales de innovación, espacio meso-económico, actores intermediarios y cooperativas: elementos teóricos y metodológicos}

De los sistemas de producción a los sistemas de innovación: el necesario empuje colectivo

Los clusters, o sistemas territoriales de producción (STP, en adelante), y entre ellos, los sistemas agroalimentarios, se enfrentan al reto de convertirse en sistemas territoriales de pro-

1. R.D. 550/2014, sobre Entidades Asociativas Prioritarias, previsto en la Ley $13 / 2013$, de 2 de agosto, de fomento de la integración de cooperativas y de otras entidades asociativas de carácter agroalimentario. Esta medida tiene por objetivos últimos "(...) el desarrollo de la innovación y la incorporación de nuevas tecnologías, aumentará su productividad y eficiencia y mejorará su capacidad de competir", siendo crucial la innovación.

2. ANECOOP se crea en 1975 y se constituye formalmente como cooperativa agraria de $2^{\circ}$ grado en junio de 1977 con la denominación de Sociedad Cooperativa de Exportación de Frutos Cítricos - Anecoop. 
ducción y de innovación (STPI, en adelante). Durante mucho tiempo los STP basaron su dinamismo en un aprendizaje interactivo informal que ha generado un importante saberhacer productivo-comercial de carácter tácito. Este proceso fue conducido por un sistema de pequeñas y medianas empresas de origen endógeno apoyado en instituciones informales que obtienen del territorio la proximidad necesaria para generar confianza y cooperación entre actores (Piore y Sabel, 1984; Becattini, 1994; Torre y Wallet, 2013).

Con la globalización, estos STP se enfrentan al reto de desarrollar formas de aprendizaje y de cooperación más sistemáticas y formales para poder generar ventajas competitivas sostenibles basadas en la capacidad de innovación (Carbonara, 2004). Pero a muchas pymes de los distritos industriales y STP en general de países desarrollados les resulta muy difícil desarrollar las competencias necesarias para innovar interna y sostenidamente y para urdir una trama de relaciones formales e informales con otras empresas, instituciones científico-tecnológicas y otras entidades del entorno peri-productivo (Carbonara, 2004; Corò y Micelli, 2007; Caniels y Van den Bosch, 2011; Temblay et al., 2012). En especial, estos entramados de actores empresariales se revelan con frecuencia incapaces de encauzar un proceso de acción colectiva más planificado y formalizado que permita responder a las nuevas exigencias. En este contexto, suele atribuirse a la existencia en el territorio de instituciones intermedias o actores intermediarios, tales como empresas líderes y asociaciones empresariales, un papel determinante en la capacidad de adaptación de los distritos industriales (Carbonara, 2004; Dei Ottati, 2003; Corò y Micelli, 2007; Temblay et al., 2012; Zeitlin, 2008) al apoyar a las empresas y propiciar su cooperación y acción colectiva.

El cometido de estos agentes intermediarios trasciende sus funciones estatutarias, si se les analiza desde la perspectiva sistémica. En efecto, un doble cometido potencial puede serle identificado: de un lado, su papel de impulso al cambio radical y, de otro lado, su papel de apoyo a la política de innovación. Ambos cometidos exigen que el agente intermediario ocupe una posición estratégica en el seno del sistema productivo-empresarial, con capacidad para impulsar la transformación de este sistema, y que al propio tiempo tenga capacidad para incidir sobre el entorno de dicho sistema generando y catalizando mecanismos de apoyo en el mismo. La base para esta argumentación es la siguiente. En contextos de clusters caracterizados por una acusada heterogeneidad empresarial, es decir, donde las diferencias de competencias entre las empresas que los conforman son muy grandes (Tomás Carpi et al., 1999; Corò y Micelli, 2007), es poco probable que pueda producirse el salto requerido en el proceso de innovación en ausencia de un cambio colectivo en la visión y la estrategia empresarial del conjunto del colectivo. $Y$ esto requiere precisamente de la existencia de algún actor del sistema que simultáneamente pueda jugar el papel de líder/tractor, implicando en el proyecto colectivo a los demás miembros del sistema, y que, al propio tiempo, pueda prestar servicios a las empresas para que eleven su capacidad de absorción de las innovaciones.

Este planteamiento amplía la teoría de las empresas líderes como actores intermediarios en la modernización de los clusters o distritos industriales (Tomás Carpi et al., 1999; Corò y Micelli, 2007) pero desde una conceptualización distinta y poniendo énfasis en las limitaciones de las empresas privadas al uso. Se sostiene que la heterogeneidad empresarial en el seno de los STP hace que dicho liderazgo sea vital para conseguir elevar de forma colectiva el nivel de competencias del conjunto de empresas.

Respecto a la segunda cuestión señalada, la del apoyo a la política de innovación, es sabido que la política pública requiere, para ser 
efectiva, de mecanismos de transmisión de sus impulsos y de un feed-back de las actuaciones que permita su renovación y actualización constantes, en un proceso de co-producción tanto de la elaboración como de la aplicación de dichas políticas. Proceso de coproducción en el que participan, y se implican, los diferentes actores del sistema, públicos y privados, empresas, universidades y otros agentes. En este contexto, el tipo de agentes intermediarios elegido por los poderes públicos resulta estratégico para que pueda estructurarse la acción de la política de innovación en el ámbito de los STP, pero también el entorno institucional en el que se alojan las relaciones inter-actores. En suma, el tránsito de STP hacia STPI va a requerir a menudo del desarrollo de un emprendimiento colectivo que impulse el proceso de innovación, al tiempo que sirva de apoyo a la política pública de innovación, lo que desborda la consideración única de los actores públicos y de las asociaciones empresariales en la política de innovación y de modernización de los distritos y STP en general (Werker y Athreye, 2004; Corò y Micelli, 2007).

Innovación como cambio en las meso-reglas

El enfoque de partida es heredero de la aportación de Schumpeter de 1939, según el cual la base del progreso económico (y de la innovación) se encuentra en la creación de nuevas reglas, esto es, combinaciones inéditas de elementos de producción, distribución y consumo por parte de los actores, las cuales generan nuevos paradigmas económicos (Schumpeter, 2002). Para que esa innovación (nueva combinación, regla o rutina) sea efectiva ha de ser reconocida, aceptada y adoptada por un número significativo de agentes del sistema. Nelson y Winter (1982) actualizaron esta idea schumpeteriana indicando que, cuando los actores operan en un contexto de incertidumbre radical y racionalidad limitada, las empresas se comportan si- guiendo rutinas o reglas organizativas. Dichas rutinas presentan una doble dimensión procedimental y substantiva. La primera enfatiza la conformación de las rutinas por prueba y error a través de un proceso espontáneo de descubrimiento de una cultura general de la organización. Esta es la supuesta base del dinamismo de los distritos industriales tradicionales y los STP (Carbonara, 2004; Corò y Micelli, 2007). Sin embargo, como la empresa y otras organizaciones reúnen bajo una misma estructura a individuos dotados de capacidades y de intereses que pueden ser divergentes $o$ incluso conflictivos, la figura del empresario o del liderazgo público es esencial para proporcionar una visión y una estrategia a estas organizaciones que juegue un papel integrador en las mismas. Esta es la perspectiva substancialista de la racionalidad (Witt, 1998). De este modo, la imaginación de los emprendedores $y$, por consiguiente, su capacidad para proyectar una visión y una estrategia, es esencial en el cambio económico en general (Witt, 1998) y en el sector agroalimentario en particular (Knudson et al., 2004). La imaginación espolea el desarrollo del conocimiento y éste es el motor del proceso de evolución (Foster y Metcalfe, 2012).

Las iniciativas empresariales o emprendimiento son los motores del cambio y la transformación porque provocan un cambio colectivo "no en una, sino en un rango de reglas de comportamiento" que son adoptadas y aplicadas por los actores económicos que toman las decisiones cuando se enfrentan a la incertidumbre (Foster y Metcalfe, 2012). Estas reglas de comportamiento no se definen en el plano microeconómico sino meso-económico porque son el resultado de una diversidad de comportamientos interactivos que generan nuevas reglas generales que se imponen a las reglas específicas de cada organización.

En este sentido, lo que provoca el cambio es el desarrollo del conocimiento en el ámbito meso-económico vía "creación, adopción, adaptación y difusión de reglas en algún sen- 
tido genéricas" (Dopfer et al., 2004). Estas meso-reglas genéricas están conformadas por nuevas prácticas tecnológicas, organizativas e institucionales articuladas entre ellas (Dopfer et al., 2004). En virtud de este planteamiento, que se preocupa de explicar el cambio y la coordinación en un sistema económico, el nivel meso no es entendido como un nivel intermedio entre los niveles micro y macro, sino el ámbito en el que se produce el cambio en las reglas $y$, por consiguiente, determinante tanto de lo micro como de lo macro.

Rutinas, redes, comunidades de práctica y epistémicas

El emprendimiento y el desarrollo de ideas no presentan únicamente un carácter individual y vinculado a la creación de nuevas empresas, sino que también operan en el plano mesoeconómico, porque comportan un proceso de coordinación dentro de la empresa y en el desarrollo de proyectos empresariales colectivos. En este sentido, Cohendet y Llerena (2003) consideran que existe un nivel intermedio de rutinas (en la empresa), entre los individuos y la organización (empresa), que es la comunidad. Las empresas se insertan en diferentes tipos de comunidades, entre las que destacan las comunidades de práctica y las comunidades epistémicas. Una comunidad de práctica ( $\mathrm{CP}$, en adelante) es un conjunto de personas que comparten una serie de tareas y de reglas formales e informales para desarrollarlas. Una comunidad epistémica (CE, en adelante) es una comunidad de práctica cuyos miembros están volcados en hacer avanzar una determinada área del conocimiento con un determinado enfoque científico. El empresario coordina las comunidades de la empresa según su visión. Ahora bien, estas comunidades pueden desbordar las fronteras de las empresas individuales (Cohendet y Llerena, 2003; Wenger, 2001; Dunlop, 2013). Así, estas comunidades también operan y se definen en las relaciones entre diferentes colectivos de diferentes empresas y en las rela- ciones entre empresas y otros actores, en particular del ámbito científico-tecnológico. Las CP y las CE constituyen así un espacio de conformación y de cambio de las rutinas en el plano meso-económico. Esto nos permite conceptualizar la articulación del nivel meso-económico vía coordinación entre diferentes actores, comunidades, etc.

Partimos de que para pasar de STP a STPI, las empresas requieren de nuevas prácticas que exigen mayores dosis de conocimiento científico. Y ello obliga a la rearticulación entre CP y CE. Cooke (2003) propone dos modelos de transición: el Entrepreneurial Regional Innovation System, liderado por empresas privadas, y los Institutional Regional Innovation Systems, liderado por la iniciativa pública en ausencia del empuje empresarial privado. Nosotros contemplamos un tercer modelo, hibrido, liderado por las cooperativas, cuya virtud radica en la conciliación público-privado, impulsando la innovación de mercado y propiciando su difusión vía política pública.

Cooke (2003) apunta diversos actores intermediarios tecnológicos para la integración de CP y CE. Ahora bien, desde el momento en que adoptamos una perspectiva substancialista de las rutinas y de la racionalidad, otros instrumentos de actuación devienen esenciales para conformar STPI. Por ejemplo, las asociaciones de empresarios, que pueden contribuir al establecimiento de relaciones entre empresas (y CP) y centros tecnológicos y universidades (y CE), etc. De igual modo, las estructuras de segundo grado de las cooperativas pueden jugar un papel esencial en priorizar ciertas rutinas frente a otras y pueden ser vitales en el impulso de una visión y estrategia empresarial compartida por las cooperativas de base. En este contexto, y a partir de la fundamentación desarrollada más arriba, podemos atribuir a las cooperativas de segundo grado un papel de collective entrepreneurship, que devendría esencial para la efectiva conformación de los Cooperative Regional Innovation Systems. 
El paso de STP a STPI o cómo articular CP y CE

Los procesos de cambio productivo, organizativo y comercial en el STP generan nuevos procesos de aprendizaje. Como las CP y las CE desbordan el ámbito de la empresa, resulta crucial generar un proceso de socialización interorganizacional para desarrollar este aprendizaje. Desde esta óptica, las interacciones entre empresas y entre firmas y centros tecnológicos y universidades son una condición necesaria para desarrollar estrategias innovadoras pero no una condición suficiente para desencadenar la transformación que supone pasar de un STP a un STPI en ausencia de mecanismos de institucionalización de estas interacciones. La razón es que las innovaciones en empresas, centros tecnológicos, etc., en el plano meso-económico, no provocan por ellas mismas un cambio en la meso-regla. Este cambio requiere desarrollar nuevas $C P$, nuevas CE y nuevas formas de articulación entre ellas. Al desarrollar estas nuevas comunidades se crean nuevos conocimientos y prácticas productivas, vía la constitución de nuevas redes de empresas, de centros de formación y de investigación y de unas y de otras. La incorporación a estas redes permite a las empresas acceder a las innovaciones. EI desarrollo de CP y CE es pues necesario para que las innovaciones se difundan suficientemente y se conviertan en meso-reglas.

\section{El Cooperative Regional Innovation System: las cooperativas como impulsoras del emprendimiento colectivo y como vehículo de la política de innovación en sistemas agroalimentarios}

Las cooperativas pueden constituir un actor de primer rango para apoyar la transformación de los sistemas agroalimentarios desempeñando un doble papel: por un lado, el ser el motor del emprendimiento colectivo necesa- rio para conformar una visión y una estrategia colectivas, condición de los procesos de innovación sostenidos y, por otro lado, por ser potencialmente un vehículo privilegiado de la política pública de innovación. Ambas potencialidades derivan de características genuinas propias de las cooperativas. Por un lado, de su forma de gobierno empresarial, participativa y cauce natural para la implicación de sus asociados en su proyecto y que se transmite en su original estructuración vertical con formas integradoras de primer, segundo y ulterior nivel (Spear, 2004). En segundo lugar, esa potencial capacidad de emprendimiento colectivo deriva de su naturaleza institucional, de los valores en que se inscribe (democracia, ayuda mutua, cooperación entre cooperativas, etc.,) y de su vínculo con la comunidad que hace que definan un entramado muy propicio al desarrollo del capital social en el sentido de Putnam (Spear, 2001). Todo ello redunda en una mayor capacidad de cooperación empresarial intra e intersectorial, así como de cooperación con los actores del sistema de formación e investigación.

Si partimos de que las meso-reglas constituyen el elemento esencial del proceso de coordinación y de cambio, se sostiene que las cooperativas son organizaciones apropiadas para ayudar a generar meso-reglas genéricas en amplios espacios intra e interterritoriales, en dos sentidos esenciales (Gallego, 2008): a) al compartir valores y rutinas conforman un terreno fértil para la difusión de prácticas similares dentro de una misma organización; b) al integrar a diferentes unidades económicas en una misma organización, son un mecanismo esencial para la difusión de innovaciones top-down y bottom-up dentro de la misma. Además, su fuerte implantación territorial y la obligación de comercialización del producto de sus asociados (Gallego, 2008), hacen que las cooperativas tengan propensión a ejercer la voz (en el sentido de Hirschman, 1970), lo que se traduce en una original combinación de presiones y de ayudas 
a sus asociados para que introduzcan innovaciones. Las cooperativas pueden propiciar así la conectividad, la proximidad geográfica y organizada (tecnológica, organizativa, institucional y cultural) (Torre y Wallet, 2013) en el interior de la organización cooperativa, al tiempo que espolear la elevación de las competencias de sus socios.

La imaginación, la visión y la estrategia de los directivos de las cooperativas de segundo grado pueden impulsar el cambio en la población de cooperativas que integran la organización cual proceso de emprendimiento colectivo. La organización territorial puede permitir perfectamente a las cooperativas integrarse horizontal y verticalmente siguiendo una meso-regla genérica al tiempo que cada organización o unidad micro interpreta la meso-regla según sus propias especificidades. Las cooperativas provocan cambios en las meso-reglas porque al ser capaces de difundir nuevas buenas prácticas entre sus socios mejoran su competitividad y presionan sobre los actores privados del territorio para que las introduzcan.

Ahora bien, la distancia cognitiva y la desigual capacidad de absorción entre cooperativas puede ser tan grande que no permita un proceso directo de imitación y de emulación entre las mismas, aun cuando dispongan de un entorno creativo rico en capital social (Laursen et al., 2012). Es aquí donde las cooperativas de segundo grado y los grupos cooperativos adquieren una importancia estratégica en el impulso al cambio del sistema empresarial territorial. Al tiempo que presionan a la adopción de innovaciones por las cooperativas de base, tienen la capacidad de absorción y las relaciones necesarias para poder interaccionar sistemáticamente con las universidades, centros tecnológicos, etc. A través de este doble proceso, las cooperativas de segundo grado estimulan la renovación y rearticulación de las $\mathrm{CP}$ y CE con participación de las cooperativas, la inserción de forma más compleja de las cooperativas en estas comunidades y la introducción intencional o sobrevenida de nuevas formas de interrelación entre diferentes CP. Todos estos vectores convierten a las cooperativas en una plataforma potencial para la política de innovación y para la estructuración de los sistemas de innovación en los sistemas agroalimentarios.

\section{Cambio en el sistema citrícola de la Comunitat Valenciana (España) impulsado por las cooperativas: emprendimiento colectivo y política de innovación}

Metodología y fuentes de información

En trabajos previos (Gallego, 2008 y 2009) se ha analizado el proceso de conformación del entramado de actores públicos, privados y mixtos que definen el STPI de la citricultura valenciana. El objetivo ahora es contrastar el papel esencial de la interacción de las CP y de las CE en la generación y difusión de las meso-reglas en el sistema citrícola valenciano y el rol esencial de la cooperativa ANECOOP en este proceso de innovación. Se pretende explicar cómo la necesidad de incorporar mayores dosis de conocimiento científico-tecnológico en la cadena de valor citrícola ha obligado a los actores a definir nuevas formas y espacios de interacción. El foco del análisis se sitúa en los actores y en las relaciones del sistema.

La información empírica de este estudio procede de una investigación longitudinal basada en la realización de un total de 218 entrevistas en profundidad, de las cuales 151 (el $69 \%$ ) fueron realizadas en la Comunitat Valenciana y 67 (el 31\%) en Cataluña. Las entrevistas han sido realizadas por los autores en los periodos 2007-2011 y 2013. Las entrevistas abarcan varios periodos porque se han ido estudiando de forma secuencial diversos ámbitos relacionales de un sistema de pro- 
ducción y de innovación. Por este motivo, el estudio tiene un carácter longitudinal no tanto por entrevistar a las mismas personas en diferentes periodos del tiempo (lo que sólo ha ocurrido con el $6 \%$ de los entrevistados) como por entrevistar a los mismos actores de forma repetida para estudiar diversos ámbitos del sistema de innovación. Por tanto, se ha entrevistado a 205 personas distintas. Se ha entrevistado a agricultores (56\%), centrales citrícolas privadas y cooperativas (16\%) así como a empresas proveedoras (18\%) de la cadena de valor (viveristas, proveedores de maquinaria y de productos químicos pre y post- cosecha y empresas de servicios a los agricultores) y a todas las entidades integrantes de la Red de Cítricos de la Comunitat Valenciana del IVIA (Instituto Valenciano de Investigaciones Agrarias) (10\%). Esta última está formada por investigadores de universidades, centros tecnológicos y de investigación (con inclusión de los departamentos de I+D de la cooperativa de segundo grado ANECOOP), responsables técnicos de la Conselleria d'Agricultura y representantes de asociaciones empresariales y profesionales. Las entrevistas a agricultores, gerentes y directivos de empresas han sido realizadas con cuestionarios cerrados. Por el contrario, los cuestionarios para los miembros de la Red de Cítricos eran abiertos, aunque incluían algunas preguntas cerradas similares a las formuladas a las empresas a efectos de comparación. Las entrevistas perseguían obtener información cualitativa sobre los procesos de innovación en el seno de las diferentes organizaciones y la naturaleza de las relaciones entre empresas y otras entidades del sistema institucional científico-tecnológico, así como entidades asociativas en las que se integran diversos actores.

El $46 \%$ de los 123 agricultores entrevistados son socios de una cooperativa de comercialización. Las cooperativas entrevistadas, prácticamente en su totalidad socias de ANECOOP, representan el $47 \%$ del total de centrales citrícolas entrevistadas. Al $50 \%$ de las coope- rativas, las más dinámicas, se ha accedido siguiendo el consejo experto de un directivo de ANECOOP. El resto se han elegido para tener una muestra representativa de las diferentes áreas geográficas. Tanto en el caso de las cooperativas como en el del resto de colectivos, se ha accedido a las personas entrevistadas a través de unos 10 informadores clave (que en un $70 \%$ de casos también han sido entrevistados) que son buenos conocedores de las diferentes zonas y/o colectivos objetivo y que nos han proporcionado los contactos. En el resto de casos, en torno a un $30 \%$, se ha contactado con los entrevistados a través de las propias relaciones y conocimiento personal de los autores o vía la concertación directa de la entrevista sin que mediara ninguna relación previa. Las muestras de todos los colectivos se han seleccionado privilegiando los comportamientos más dinámicos pero asegurándonos de que recogiesen la diversidad existente dentro de la población de referencia. De las entrevistas se obtienen resultados cualitativos que han sido movilizados siguiendo el programa F4 para la escucha y transcripción de las grabaciones y el programa Atlas.ti para su tratamiento, en especial en las entrevistas realizadas a los actores de la referida Red de Cítricos, cuyos actores localizados mayoritariamente en la Comunitat Valenciana constituyen el núcleo del objeto de estudio del artículo.

\section{Resultados}

Breve caracterización del sistema citrícola

Con una producción de 5.501.500 toneladas, España ocupa el sexto lugar en la producción mundial de cítricos con el 4,19\% del total, en 2012 (FAO, 2014), pero ostenta la primera posición en las exportaciones mundiales de cítricos en fresco con 3.588.400 toneladas que suponen el $27,79 \%$ del total mundial en 2010-2011 (FAO, 2012). Destaca la elevada 
cuota de mercado española en las exportaciones mundiales de mandarinas, que tradicionalmente se sitúa en torno al $50 \%$ y que alcanzaba el 38,05\% en 2010-2011 (FAO, 2012). Este gran peso de las mandarinas, se desprende de la especialización en estos cítricos de la Comunitat Valenciana, que aporta en 2011 1.572.569 toneladas, lo que supone el $74,3 \%$ de la producción española de mandarinas (MAGRAMA, 2013). Esta especialización está en la base del desarrollo de un sector líder a nivel mundial.

En el sector citrícola valenciano, las cooperativas ocupan una posición central, destacando entre ellas el sistema empresarial liderado por la cooperativa de segundo grado ANECOOP, la más grande cooperativa agraria exportadora de España, la primera empresa exportadora de productos citrícolas del Mediterráneo y la segunda del mundo. Integra actualmente 79 cooperativas asociadas con una facturación en 2011 de 438,6 millones de $€$ y 337,7 millones de $€$ de exportación (Meliá y Marí, 2013; Campos et al., 2012; Arcas y Hernández, 2013). El sector de cítricos representa el $40,2 \%$ y el $42,2 \%$ del volumen y del valor total, respectivamente, de la actividad de ANECOOP en la campaña 2011-2012 (ANECOOP, 2013).

En la Comunitat Valenciana el sector citrícola está organizado en torno a centrales citrícolas. Son cooperativas o empresas privadas, normalmente de capital valenciano y de carácter familiar, que adquieren la producción a los agricultores y se encargan de su acondicionamiento y comercialización. Han hecho tradicionalmente de cadena de transmisión de los impulsos del mercado a los agricultores. Para realizar este proceso de acondicionamiento, estas centrales se apoyan en proveedores de maquinaria y otros productos químicos de postcosecha, envases, etc. También están presentes en la Comunitat Valenciana los proveedores de inputs para la producción agraria, tales como plantones, productos químicos y maquinaria (Gallego, 2009).
El sistema agroalimentario citrícola ha sufrido en las dos últimas décadas un profundo cambio en los canales de comercialización y en las exigencias de los mercados. Las centrales citrícolas han pasado de vender mayoritariamente a mercados centrales sobre los que ejercían un cierto control e información, a comercializar el grueso de la producción a través de grandes cadenas de distribución que fijan las condiciones de venta. Además, se han introducido nuevas normas y estándares públicos y privados en lo relativo a las condiciones de producción, de tratamiento y de comercialización de la fruta, que han exigido la modernización científico-tecnológica y la reglamentación del sector. Esto ha impelido a la profesionalización de la gestión técnica y gerencial de las explotaciones y de las empresas de acondicionamiento y comercialización de la fruta (Gallego, 2009).

Cambios en la organización de la comercialización y cambios en las exigencias públicas y privadas de los productos y procesos productivos

Las nuevas exigencias de los mercados y de las propias administraciones públicas desde la década de los años 90 del siglo pasado, han llevado a un proceso paralelo de desarrollo de nuevas CP y de nuevas CE en la citricultura valenciana. De una parte, dichas exigencias han implicado la necesidad de incorporar a titulados universitarios, en especial ingenieros agrónomos, en las centrales citrícolas, tanto para controlar el acondicionamiento de los productos para su comercialización como para controlar las condiciones del cultivo de los agricultores socios. Así, a partir de los 90 adquieren un gran desarrollo las Agrupaciones para el Tratamiento Integrado en la Agricultura (ATRIAS) y las Asociaciones de Defensa Vegetal (ADV). Reguladas por el Ministerio de Agricultura (gobierno central) y por la Conselleria de Agricultura (gobiernos 
regionales), respectivamente, consisten básicamente en ayudas públicas a empresas y entidades (que mayoritariamente son cooperativas) para la contratación de técnicos y productos destinados esencialmente a la lucha contra plagas y enfermedades mediante métodos respetuosos con el medio ambiente y la salud humana. Al propio tiempo, y de otra parte, las nuevas exigencias han llevado a reorientar la investigación, para atender al nuevo concepto de calidad (más atento a la presentación del producto que a sus propiedades organolépticas), nuevas variedades y patrones, etc. En este ámbito, el cambio de paradigma ( $y$, por consiguiente, de la episteme) está asociado a la introducción de la genética (Navarro y Juárez, 2000). De igual modo, en el ámbito de la lucha contra plagas y enfermedades se asiste a un cambio radical con el paso de la lucha química a la lucha biológica (Laborda, 2012). Estos procesos han implicado una ampliación y un cambio en los equipos y en la forma de abordar la investigación en estos ámbitos especialmente por parte de la Universidad Politécnica de Valencia (UPV), así como el IVIA y la colaboración de ambas instituciones.

Respuesta cooperativa a los cambios y rearticulación de las $\mathrm{CP}$ y las CE

En este apartado se analizan tres espacios paradigmáticos de interacción entre $\mathrm{CP}$ y $\mathrm{CE}$ construidos por los actores, los cuales ejemplifican sendas formas y niveles de integración entre $\mathrm{CP}$ y $\mathrm{CE}$, de inserción en el sistema de innovación y ponen de relieve el rol clave de las cooperativas de segundo grado. Dichos espacios son los siguientes:

Ámbito 1. Relación entre técnicos del Departamento de Calidad y Sistemas de ANECOOP y técnicos de las cooperativas asociadas. Desarrollo en complejidad de las CP de los titulados universitarios incorporados al sector.
Ámbito 2. Relación entre investigadores y técnicos (en cultivos, ATRIAS, etc.) en proyectos de investigación y formación. Interacción e integración bilateral entre CP y CE.

Ámbito 3. Grupo nacional de trabajo de cítricos. Relación entre técnicos de administraciones públicas, investigadores y técnicos de empresas de la cadena de valor. Integración multilateral de CP y CE.

Ámbito 1. Relación entre técnicos del Departamento de Calidad y Sistemas de ANECOOP y técnicos de las cooperativas asociadas

Las cooperativas citrícolas valencianas presentan un pequeño tamaño. Por ello, para tratar de capturar economías de escala, ganar en capacidad innovadora y poder responder a las exigencias de los mercados (Filippi y Triboulet, 2006), han avanzado en un doble proceso de concentración horizontal y vertical (Julià et al., 2012; Gallego, 2008; Meliá y Marí, 2013; Arcas y Hernández, 2013; Giagnocavo et al., 2014). Como ya se ha avanzado al comienzo del artículo, el principal hito en este proceso lo constituye la fundación de ANECOOP en 1975 y su constitución en 1977 como cooperativa de segundo grado en el ámbito de la comercialización dirigida a la exportación. Un proceso que comienza con 31 cooperativas valencianas en torno a la exportación de cítricos, pero que irá incorporando a nuevas regiones y cooperativas españolas (Font de Mora, 2003). Las cooperativas asociadas se comprometen a comercializar un porcentaje mínimo de su producción a través de ANECOOP, siendo el porcentaje medio de entrega real en la actualidad el 62\% (Meliá y Marí, 2013), lo que da una idea de su capacidad de integración cooperativa. ANECOOP ha estimulado también la fusión de las cooperativas de base y la propia fusión de su gestión con mantenimiento de la independencia de las mismas (Planells y Mir, 2004: 
397; Meliá y Marí, 2013). Este proceso ha avanzado desde principios del nuevo siglo con la creación de un Grupo Cooperativo que supone un mayor grado de integración entre las cooperativas que lo integran (ANECOOP, 2013).

Ahora bien, en ANECOOP coexisten algunas centrales citrícolas líderes mundiales con pequeñas centrales con problemas de profesionalización técnica y gerencial. Un reto esencial para la dirección de ANECOOP es lograr que la producción que les suministran unas cooperativas tan heterogéneas reúna las condiciones de calidad, seguridad alimentaria y respeto al medio ambiente que le exigen sus clientes. Para poder ayudar a las cooperativas de base, ANECOOP ha estimulado en el ámbito productivo-comercial un cambio en el conocimiento base del sector (regla genérica). Este proceso se apoya, en primer lugar, en una mejora de sus competencias internas de investigación, tanto en materia de postcosecha (Departamento de Calidad y Sistemas) como en el ámbito de la producción (Departamento de Producción y Desarrollo). Esto le ha permitido estrechar sus lazos con proveedores y firmar convenios de investigación con importantes centros de investigación locales, tales como el IATA (Instituto de Agroquímica y Tecnología de Alimentos, CSIC).

En segundo lugar, ANECOOP obliga a las cooperativas de base a adoptar sustanciales mejoras tecnológicas y organizativas para poder permanecer dentro de la organización y les ayuda a responder a estas exigencias. En efecto, desde el Departamento de Calidad y Sistemas de ANECOOP se despliega una labor de difusión de los resultados de la investigación y de asesoramiento técnico a las cooperativas de base, lo cual se concreta en tres elementos (Gallego, 2008; ANECOOP, 2013): a) reuniones con técnicos de las cooperativas de primer grado; b) establecimiento de protocolos y un sistema propio de buenas prácticas en almacén y en el ám- bito productivo (producción integrada) y c) visitas a las centrales citrícolas de las cooperativas por un equipo de técnicos para fiscalizar y asesorar sobre buenas prácticas.

Estos técnicos de ANECOOP se convierten en el vehículo de la visión y estrategia de la organización de segundo grado en lo relativo a cómo se relaciona toda la organización con el entorno (mercadológico y tecnológico), impulsando un proceso de selección interna de rutinas. Junto a esta labor de formación y de difusión de innovación técnica desde ANECOOP a las cooperativas de base, también se encuentra una labor de formación sociocultural que es esencial para lograr una "progresiva mayor implicación del socio con las cooperativas" (Chaves, 2003). Esto último, unido al reforzamiento de la interacción con ANECOOP de los directivos y consejos rectores de las cooperativas asociadas a ANECOOP, es esencial para intensificar el sentido de pertenencia, aceptar las reformas estructurales en las cooperativas (Meliá y Marí, 2013) e interiorizar la visión y estrategia de ANECOOP.

\section{Ámbito 2. Relación entre investigadores y técnicos en proyectos de investigación y formación}

Buena parte de la investigación de ANECOOP con universidades y centros de investigación se realiza en las propias centrales citrícolas de las cooperativas, dado que ANECOOP cuenta con campos de experiencias pero carece de instalaciones propias de acondicionamiento de la fruta. Este proceso también tiene lugar en el ámbito de la producción, ya que a) las cooperativas de base se responsabilizan directamente de la calidad, seguridad alimentaria y respeto medio ambiental de la producción de los socios (agricultores), lo que supone que van a contar con la mayoría de los técnicos de las ATRIAS y ADVS y además b) las cooperativas poseen los principales campos de experimentación del sector (Gallego, 2008). Todo esto ha generado una re- 
lación interactiva y sinérgica entre los técnicos y los investigadores gracias al carácter aplicado de la investigación en múltiples campos (plagas y enfermedades, fisiología vegetal, etc.), en los que se dirime la calidad, la seguridad alimentaria y el medio ambiente. De este modo, no sólo los técnicos requieren de la interacción con los científicos, sino que en el avance del conocimiento de estos últimos es esencial contar con una red de campos de experimentación y de técnicos de elevado nivel, para poder experimentar nuevos productos y técnicas de cultivo y de manejo contra plagas, etc., y para poder tener un feed-back de los mismos. Relación simbiótica, que comporta una creciente integración entre las $\mathrm{CP}$ y las $\mathrm{CE}$, y que es además esencial en la generación de relaciones informales estables entre investigadores y técnicos de las cooperativas (Gallego, 2009).

Esta creciente proximidad entre investigadores y técnicos se refuerza con la formación. Por ejemplo, desde 1989 la Federación de Cooperativas Agrarias de la Comunitat Valenciana (FECOAV) viene realizando un curso de producción integrada para técnicos con conocimientos de agronomía de las cooperativas, impartido por expertos de la universidad, centros de investigación y administración pública. Este curso anual se revela un foro esencial para la interacción sistemática y la generación de confianza entre técnicos del sector, profesores de universidad y expertos y responsables en la materia en las administraciones públicas y, por tanto, un vivero de capital social.

Ámbito 3. Grupo nacional de trabajo de cítricos. Relación entre investigadores, técnicos de administraciones públicas y empresas de la cadena de valor

Los grupos nacionales de trabajo en diversos cultivos fueron creados por el Ministerio de Agricultura en los años 70 para coordinar a las diferentes regiones españolas en materia de plagas y enfermedades. El Ministerio re- gula y coordina en este ámbito y las Comunidades Autónomas tienen la competencia y la experiencia en la aplicación de los conocimientos y las normas legales. Estos grupos integran tres tipos de actores. Primero, representantes de las administraciones públicas, estatal y autonómica. Suelen ser mandos intermedios de perfil técnico. Un segundo actor está formado por expertos investigadores y técnicos de las ATRIAS, normalmente de las cooperativas. En el caso del grupo de trabajo de cítricos, todos los investigadores valencianos proceden del IVIA o de la UPV. En tercer lugar, también participan puntualmente técnicos y/o comerciales de las empresas de productos fitosanitarios. El grupo de cítricos está formado por unas veinte personas, de las que la Comunitat Valenciana aporta una cuarta parte dado su peso en el sector citrícola. Los grupos hacen recomendaciones de productos para la lucha contra plagas y enfermedades. También fijan reglas y estándares, por ejemplo en lo relativo a la producción integrada. Sus decisiones no son legalmente vinculantes, pero sí vinculan moralmente por la forma en que se alcanzan.

En las reuniones anuales, los grupos se reparten los trabajos a realizar entre los técnicos de la administración y de las ATRIAS y los investigadores de las diferentes regiones. Es decir, la verificación de la eficacia, respeto al medioambiente y a la salud de las personas de los nuevos productos fitosanitarios que aspiran a ser registrados, así como la realización de ensayos sobre la eficacia de diversos productos y sobre técnicas de manejo novedosas, tales como la confusión sexual. A partir de este trabajo, el grupo realiza sus recomendaciones tanto en el ámbito del registro de producto como en los Boletines de Avisos de las comunidades autónomas. Esta labor en momentos de normalidad se combina con una labor excepcional como gabinete de crisis cuando surge una nueva plaga desconocida, estableciendo la forma de afrontarla y la orientación subsiguiente de la investigación. 
Los resultados de los grupos de trabajo contribuyen a formar la posición nacional que defiende la administración central española en las instituciones internacionales, al tiempo que legitiman una perspectiva epistémica y un enfoque de manejo contra las plagas y enfermedades en las instituciones internacionales. Esta gran influencia de estos grupos de trabajo proviene de que en su interior se genera una fuerte integración multilateral entre $C P$ y $C E$, en el sentido de que se ven involucrados no únicamente los técnicos de las cooperativas y de los centros de formación y de investigación, sino también de otros subsectores de la cadena de valor y. sobre todo, las propias administraciones públicas, central y autonómica y, por consiguiente, la política de innovación.

Hay que tener en cuenta que la inmensa mayoría de técnicos e investigadores participantes son ingenieros agrónomos, de forma que comparten la misma episteme al tiempo que han mantenido fuertes relaciones profesor-alumno, todo lo cual contribuye a la cohesión, confianza y proximidad cognitiva entre ellos que favorece la cooperación. Máxime teniendo presente que, como nos indica un miembro del Grupo de Cítricos "dentro de los ingenieros agrónomos, la sanidad vegetal es un campo muy específico, con canales de acceso al empleo específicos y personal con larga experiencia". Ello genera una identidad propia dentro de la CP en sanidad vegetal y que desborda la frontera entre técnicos e investigadores. Esto reproduce así, sobre una base ampliada, la lógica de la interacción sinérgica y simbiótica entre técnicos (de cooperativas) e investigadores analizada en el apartado anterior.

Las cooperativas juegan un papel esencial en todo este proceso. En primer lugar, participan directamente en este grupo de cítricos y en la generación de los resultados del grupo, que pueden ser considerados innovaciones de producto (fitosanitarios) y de proceso (ma- nejo) contra plagas. En segundo lugar, contribuyen directamente a la generación de estos resultados a través de la realización en sus campos de experimentación de los ensayos del grupo. En tercer lugar, los mandos intermedios de las consejerías de agricultura organizan reuniones de coordinación con las ATRIAS (formadas por cooperativas y otras formas de organización colectivas), para transmitirles los resultados de las reuniones de los grupos. Las ATRIAS son pues una vía directa de acceso de los agricultores a los resultados de estos grupos. En definitiva, las cooperativas son esenciales tanto en la generación de innovaciones como en su difusión, con el consiguiente feed-back creativo que supone esta difusión.

\section{Discusión}

Los sistemas agroalimentarios se enfrentan al reto de pasar de ser sistemas territoriales de producción (STP) a ser sistemas territoriales de producción y de innovación (STPI). Una primera precisión conceptual que aporta el artículo en el ámbito de la literatura sobre innovación en STPI, es suponer que para tener éxito en este envite es esencial comprender que, dado que los STP albergan una gran heterogeneidad empresarial, resulta esencial que los actores intermediarios participen al propio tiempo en el sistema productivo y presenten suficiente capacidad de liderazgo para conducir, presionar, implicar y ayudar a mejorar a las empresas del resto del colectivo empresarial. Esto es lo que permite comprender y conceptuar el enfoque evolutivo micro-meso-macro que sitúa en el ámbito meso-económico el espacio donde se conforman los cambios. Ello se produce a través de un cambio en las prácticas tecnológicas, organizativas e institucionales que definen las meso-reglas genéricas. $Y$ una aportación del presente artículo es tratar de ofrecer una 
articulación conceptual entre este enfoque con el enfoque de las comunidades de práctica (CP) y comunidades epistémicas (CE), con el fin de poder representar el cambio en los sistemas agroalimentarios hacia STPI. En efecto, las meso-reglas se van a definir esencialmente a través de las $C P$ y de las $C E$, que desbordan el ámbito de las empresas (y también de otras instituciones científico-tecnológicas) y permiten una articulación entre los actores que conforman el STPI. En especial, cuando las meso-reglas suponen un nuevo tipo de rutinas y procedimientos que incorporan en mayor grado el conocimiento científico, entonces se impone la generación de nuevos conocimientos que desbordan las fronteras entre CP y CE. La visión colectiva que emana de los procesos de emprendimiento colectivo (producto de la interacción entre actores) es la que impulsa estos cambios.

$Y$ en este marco, la principal idea-fuerza del artículo es que las cooperativas de segundo y ulterior grado son capaces de impulsar un cambio en las meso-reglas gracias a su capacidad de disciplinar, implicar y ayudar a las cooperativas socias para que adopten las nuevas rutinas. Todo ello gracias al modo genuino de gobierno que les caracteriza, a su fuerte cohesión interna y al elevado capital social que destilan las cooperativas cuando despliegan efectivamente sus valores propios. Esto supone la existencia de una tercera vía (cooperativa) hacia los STPI junto a las Entrepreneurial Regional Innovation System (basado en la dinámica de los start-up) y los Institutional Regional Innovation Systems (basados en el impulso de las administraciones públicas): el Cooperative (Institutional) Regional Innovation Systems para los sistemas agroalimentarios. Este tipo de sistemas se basarían, de un lado, en el impulso empresarial de las cooperativas de segundo y ulterior grado. $Y$, de otro, en que dicho impulso y la propia organización y cohesión interna cooperativa crearían bases muy apropiadas para la im- plementación de la política pública de innovación, en la medida que proporcionaría un canal eficaz para difundir la meso-regla genérica.

Siguiendo esta perspectiva, la principal aportación empírica del artículo es haber mostrado el proceso de conformación de un modelo cooperativo de sistema de innovación a través de la organización impulsada por ANECOOP de una arquitectura institucional de relaciones interactivas de diferente grado de complejidad entre cooperativas y entre éstas y otros actores científico-tecnológicos, que constituyen el vehículo de la transformación territorial a través de la redefinición entre las CP y CE. ANECOOP ha impulsado una visión y un proceso de emprendimiento colectivo que presiona a (y eleva las competencias de) las cooperativas de base, al tiempo que les ofrece la ayuda necesaria para responder a sus exigencias. Este acicate a la mejora continua y esta garantía de comercialización en mercados exigentes que supone para los agricultores la pertenencia de sus cooperativas a ANECOOP, sugiere una dimensión esencial para comprender el comportamiento y la satisfacción de los agricultores socios de las cooperativas y que sin embargo queda fuera de los análisis en términos de racionalidad plena e individual (Arcas et al., 2013). El artículo evidencia cómo a partir del esfuerzo investigador de ANECOOP se ha desarrollado un proceso en espiral de complejidad creciente que ha supuesto el establecimiento de relaciones estables entre técnicos de cooperativas e investigadores de los centros de investigación regionales y la dinamización de instituciones multilaterales. En estas últimas las administraciones públicas encuentran no sólo las redes de la cooperativa de segundo grado para desplegar su política de innovación. También se encuentra un espacio de co-evolución y de integración entre las comunidades de práctica y las comunidades epistémicas que legitima mutuamente la posición de los actores parti- 
cipantes gracias a la generación colectiva del conocimiento, un aspecto esencial en las políticas públicas. Así el artículo cumple su objetivo empírico esencial el cual era ofrecer una vía genuinamente cooperativa del paso de un sistema territorial de producción a un sistema territorial de producción y de innovación en la citricultura valenciana.

\section{Agradecimientos}

Los autores agradecen el apoyo financiero del Ministerio de Ciencia e Innovación (MICINN) (Proyecto DER2009-14462-C02-02) y J. R. Gallego Bono agradece el apoyo financiero de la Fundación Bancaja y del LabexMed (Les sciences humaines et sociales au cœur de l'interdisciplinarité pour la Méditerranée) de la Aix-Marseille Université para realizar en 2013 una estancia de dos meses en el LEST (Laboratoire d'Économie et Sociologie du Travail) de Aix-en-Provence, así como la ayuda de la profesora Annie Lamanthe durante la misma. Los autores también agradecen las sugerencias de los evaluadores anónimos que han permitido mejorar el artículo.

\section{Bibliografía}

ANECOOP (2013). Anecoop memoria RSC 2011/2012, Valencia, Anecoop, S. Coop. Disponible en http://www.anecoop.com/ (22 marzo 2013).

Arcas N, Hernández M (2013). Tamaño y competitividad: experiencias de crecimiento en las cooperativas agroalimentarias españolas, Almería, Cajamar Caja Rural.

Arcas N, Martín JF, Mínguez A (2013). Satisfacción de los socios con las cooperativas agrarias. Una explicación a partir de la Teoría de los Derechos de Propiedad. Información Técnica Económica Agraria 109 (4): 443-457.
Becattini G (1994). El distrito marshalliano: una noción socio-económica. En: Las regiones que ganan (Eds. G Benko, A Lipietz), pp. 39-57. Valencia: Ed. Alfons el Magnànim.

Caniels MCJ, Van den Bosch H (2011). The role of higher education institutions in building regional innovation systems. Papers in Regional Science 90(2): 271-287.

Campos V, Apetrei A, Chaves R (2012). Delphi method applied to horticultural cooperatives. Management Decision 50(7): 1266-1284.

Carbonara N (2004). Innovation process within geographical clusters: A cognitive approach. Technovation 24(1): 17-28.

Chaves R (2003). El desarrollo agrario a través del cooperativismo. El caso de la cooperativa agraria Anecoop S. Coop. En: Elementos de economía social (R Chaves, JL Monzón, A Sajardo), pp.107-116. Valencia: Universidad de Valencia.

Cohendet P, Llerena P (2003). Routines and incentives: the role of communities in the firm. Industrial and Corporate Change 12 (2): 271-297.

Cooke $\mathrm{P}$ (2003). The regional development agency in the knowledge economy: boundary crossing for innovation systems. Prepared for European Regional Science Association Annual Conference - "Peripheries Centres and Social Development in the New Europe", Jyväskylä, Finlandia, 27 al 30 de Agosto.

Corò G, Micelli S (2007). Industrial districts as local systems of innovation, Working Paper, $\mathrm{N}^{\circ} 6$, Departament of Economics, Ca' Foscani University of Venice (http://admin.unive.it/media/allegato/DIP/Economia/Working_papers/Wo rking_papers_2007/WP_DSE_coro_miceIli_06_07.pdf) (Consultado 26/05/2013].

Dei Ottati G (2003). Exit, voice and the evolution of industrial districts: The case of the Post-World War II economic development of Prato. Cambridge Journal of Economics 27 (4): 501-522.

Dopfer K, Foster J, Potts J (2004). Micro-meso-macro. Journal of Evolutionary Economics 14(3): 263-279.

Dunlop CA (2013). Epistemic Communities, En: Routledge Handbook of Public Policy (Eds M 
Howlett, S Fritzen, W Xun, E Araral). Chap.8, New York Routledge.

FAO (2012). Food and Agriculture Organization Citrus Fruit Fresh and Processed Annual Statistics 2012. Disponible en http://www.fao.org/fileadmin/templates/est/COMM_MARKETS_MONITORING/Citrus/Documents/CITRUS_BULLETIN_2012.pdf (14 diciembre 2014).

FAO (2014). Food and Agriculture Organization statistical database. Disponible en http://faostat.fao.org/dafault.aspx (22 octubre 2014).

Filippi M, Triboulet P (2006). Typologie des comportements à innover des coopératives agricoles.Une étude en région Midi-Pyrénées. Économie Rurale 296: 20-38.

Font de Mora L (2003). Anecoop, primera empresa hortofrutícola del Mediterráneo. Revista Valenciana de Economía y Hacienda 7(1): 169-197.

Foster J, Metcalfe JS (2012). Economic emergence: An evolutionary economic perspective. Journal of Economic Behavior and Organization 82(2-3): 420-432.

Gallego JR (2008). Economía social y dinámica innovadora en los sistemas territoriales de producción y de innovación. Especial referencia a los sistemas agroalimentarios. CIRIEC España, Revista de Economía Pública, Social y Cooperativa 60: 7-40.

Gallego JR (2009). La articulación local-global de sistemas territoriales de producción y de innovación. Revista de Estudios Regionales 84 (1): 53-82.

Giagnocavo C, Gerez S, Campos V (2014). Paths to cooperative survival: structure, strategy and regeneration of fruit and vegetables cooperatives in Almeria and Valencia, Spain. Annals of Public and Cooperative Economics 85 (4): 617-639.

Hirschman AO (1970). Exit, voice, and loyalty: responses to decline in firms, organizations and states. Cambridge (MA): Harvard University Press.

Iliopoulos C (2013). Public policy support for agricultural cooperatives: an organizational economics approach. Annals of public and cooperative economics 84 (3): 241-252.
Julià JF, Melià E, García G (2012). Strategies developer by leading EU agrifood cooperatives in their growth models. Service business 6(1): 27-46.

Knudson W, Wysocki A, Champagne J, Peterson HC (2004). Entrepreneurship and innovation in the agri-food system. American Journal of Agricultural Economics 86 (5): 1330-1336.

Laborda R (2012). Comparación de la abundancia y biodiversidad de artrópodos auxiliares entre parcelas de cultivo ecológico y convencional, en plantaciones de cítricos, caqui y nectarina. Tesis Doctoral, Valencia. Universitat Politècnica de València. 174 pp.

Laursen K, Masciarelli F, Prencipe A (2012). Regions matter: How localized social capital affects innovation and external knowledge acquisition. Organization Science 23 (1): 177-193.

Lundvall BA (1992). National system of innovation. Toward a theory of innovation and interactive learning. London y New York.

MAGRAMA (2013). Anuario de Estadística Ministerio de Agricultura, Alimentación y Medio Ambiente 2012. Subsecretaría General Técnica, Ministerio de Agricultura, Alimentación y Medio Ambiente, 1137 pp.

Meliá E, Marí S (2013). Anecoop. Ejemplo de éxito de gestión cooperativa y global. En: Tamaño y competitividad. Experiencias de crecimiento en las cooperativas agroalimentarias españolas (Dirs N Arcas, M Hernández), pp.105-129. Almería, Cajamar Caja Rural.

Navarro L, Juárez J (2000). Aplicaciones de la biotecnología en cítricos y en otros cultivos. Comunidad Valenciana Agraria 17: 3-12.

Nelson RR, Winter S (1982). An evolutionary theory of economic change. Cambridge (MA): Harvard University Press.

Nilsson J, Van Dijk G (eds.) (1997). Strategies and structures in the agro-food industries, Assen, Van Gorcum.

Piore M, Sabel C (1984). The second industrial divide: possibilities for prosperity. New York: Basic books. 
Planells JM, Mir J (2004). Grupo empresarial Anecoop. Origen y desarrollo. En: Economía Social. La actividad económica al servicio de las personas. (Coord. JF Juliá), Mediterráneo Económico 6: 386-402.

Schumpeter JA (2002). Ciclos económicos. Análisis teórico, histórico y estadístico del proceso capitalista. Zaragoza. Prensas Universitarias de Zaragoza (V.O. 1939).

Spear R (2001). The cooperative advantage. Annals of Public and Cooperative Economics 71(4): 507-523.

Spear R (2004). El gobierno democrático en las empresas cooperativas. CIRIEC-España, revista de economía pública, social y cooperativa 48: 11-30.

Temblay DG, Klein JL, Dossou A (2012). Les acteurs intermédiaires dans le développement de l'innovation. Revue d'Économie Régionale \& Urbaine 3: 431-454.

Tomás Carpi JA, Gallego JR, Picher JV (1999). Cambio tecnológico y transformación de sistemas industriales localizados: la industria cerámica española. Información Comercial Española 781: 45-68.
Torre A, Wallet F (2013). Innovation and governance of rural territories. En: Renewing innovation systems in agriculture and food (Eds. E Coudel, H Devautour, CT Soulard, G. Faure, B Hubert), pp. 147-164, Wageningen Academic Publishers.

Wenger E (2001). Comunidades de práctica. Barcelona: Paidós.

Werker C, Athreye S (2004). Marshall's disciplines: knowledge and innovation driving regional development and growth. Journal of Evolutionary Economics 14(5): 505-523.

Witt U (1998). Imagination and leadership - The neglected dimension of an evolutionary theory of the firm. Journal of Economic Behavior and Organization 35(2): 161-177.

Zeitlin J (2008). Industrial districts and regional clusters. En: The Oxford Handbook of Business History (Eds. G Jones, J Zeitlin), pp. 219-243. Oxford University Press.

(Aceptado para publicación el 7 de febrero de 2015) 